\title{
TRACKING VARIABLE NUMBER OF TARGETS USING SEQUENTIAL MONTE CARLO METHODS
}

\author{
William Ng, Jack Li, Simon Godsill, and Jaco Vermaak \\ Department of Engineering \\ University of Cambridge \\ Trumpington Road, Cambridge, \\ U.K. \\ phone: + (44) 12233 32767, fax: + (44) 12233 32662, email: \{kfn20,jfl28, sjg, jv211\}@eng.cam.ac.uk \\ web: http://www-sigproc.eng.cam.ac.uk
}

\begin{abstract}
In this paper, we present a new approach for online joint detection and tracking for multiple targets, using sequential Monte Carlo methods. We first use an observation clustering algorithm to find some regions of interest (ROIs), and then propose to initiate a new target or remove an existing track, based on the persistence information of these ROIs over time. In addition, we also integrate a very efficient 2-D data assignment algorithm into the sampling method for the data association problem. Computer simulations demonstrate that the proposed approach is robust in performing joint detection and tracking for multiple targets even though the environment is hostile in terms of a high clutter rate and a low target detection probability.
\end{abstract}

\section{INTRODUCTION}

Online joint detection and tracking for multiple targets remains a challenging problem for surveillance systems using one or multiple sensors to monitor the environment. Typical applications can be found in navigation, air trafic control, and military surveillance systems.

Recently, sequential Monte Carlo (SMC) methods, also known as particle filters, have become popular in recursively tracking moving objects. To cope with the joint multitarget detection and tracking scenario, approaches like $[1,2]$ were proposed, but according to [3] they are not robust if the environment becomes more hostile, such as higher clutter density and low probability of target detection. In this paper, we extend the hybrid approach in [3] to a new approach in the context of a SMC framework that stochastically estimates the number of targets and hence the multitarget state. Moreover, to address the complex measurement-to-target association problem, we adopt the soft-gating approach in [4].

This paper is organised as follows. Section 2 presents a general state-space model for the problem, and a brief description of the SMC framework for joint target detection and tracking. Section 3 briefly presents the brith and death moves for estimating the number of targets. Simulation results are shown in Section 4, followed by the conclusions in Section 5.

\section{DATA MODEL}

The data models we use in this paper follow those in $[3,4]$, so we only a brief decription here. Let $\mathbf{x}_{t}$ be a combined target state vector for $K_{t}$ unknown and time-varying targets as $\mathbf{x}_{t}=\left[\mathbf{x}_{1, t}^{T}, \ldots, \mathbf{x}_{k, t}^{T}, \ldots, \mathbf{x}_{K_{t}, t}^{T}\right]^{T}$, which follows a dynamic model, given by

$$
\mathbf{x}_{k, t}=\mathbf{f}_{k}\left(\mathbf{x}_{k, t-1}, \mathbf{v}_{k, t}\right), k \in\left\{1, \ldots, K_{t}\right\},
$$

where $\mathbf{x}_{k, t}$ denotes the state vector of the $k$ th target, and $\mathbf{f}_{k}(\cdot)$, which models the motion of the target, can be a linear or nonlinear function. The noise $\mathbf{v}_{k, t}$ is assumed to be zeromean with a fixed and known covariance matrix $\boldsymbol{\Sigma}_{v}$. It is assumed that all targets are moving independently according to Markovian dynamics [5].

The number of targets $K_{t}$ is modelled by the following stochastic relationship at time $t$

$$
K_{t}=K_{t-1}+K_{t},
$$

where $K_{t}$ is a discrete iid random variable such that

$$
\begin{aligned}
\operatorname{Pr}\left(K_{t}=-1\right) & =h_{d}, \\
\operatorname{Pr}\left(K_{t}=0\right) & =1-h_{b}-h_{d}, \\
\operatorname{Pr}\left(K_{t}=1\right) & =h_{b},
\end{aligned}
$$

where $h_{b}, h_{d} \in\{0,1\}$ are the pre-defined probabilities for incrementing and decrementing the number of targets, respectively. Note that the models in (2) and (3) state that the number of targets can change by no more than one at a given time. In general, we set $h_{d}=h_{b}=h / 2$, where $h \in\{0,1\}$, but when $K_{t-1}=0$, we set $h_{d}=0$ and $h_{b}=h$.

Throughout this paper, a single sensor is employed, but the entire framework can be readily extended for multiple sensors. Let $\mathbf{y}_{t}$ be an observation received by the sensor, whose $m$ th measurement may originate from a true target or a false alarm, or otherwise known as clutter. We denote an association vector by $t=\left[\begin{array}{lll}1, t, \ldots, & m, t\end{array}, \ldots, M_{t},\right]^{T}$, where $M_{t}$ is the number of measurements. It is assumed that the measurement-to-target assignment is always on a one-to-one basis. If the $m$ th measurement $\mathbf{y}_{m, t}$ is associated with the $k$ th target, $m, t=k$, and $\mathbf{y}_{m, t}$ can be expressed as

$$
\mathbf{y}_{m, t}=\mathbf{g}\left(\mathbf{x}_{k, t}, \mathbf{w}_{m, t}\right),
$$

where $\mathbf{g}(\cdot)$, the $m$ th observation model, may be a linear or nonlinear function, $\mathbf{w}_{m, t}$, mutually independent of $\mathbf{v}_{k, t}$, is also assumed to be zero-mean with a fixed and known covariance matrix $\boldsymbol{\Sigma}_{w}$. While a true target may exist, it may not be detected when, for example, the probability of target detection $P_{D}$ is low, and its measurement may not be received by the sensor, leading to data loss. On the contrary, ${ }_{m . t}$ is set to zero if $\mathbf{y}_{m, t}$ originates from clutter, whose distribution 
is assumed uniform over the surveillance region [6, 7]. From this point onward, it is assumed that all targets share the same evolution model, and that all measurements share the same observation model.

In the context of joint detection and tracking, we are interested in estimating the posterior distribution $p\left({ }_{t} \mid \mathbf{y}_{1: t}\right)^{1}$, where $t=\left\{\mathbf{x}_{t}, K_{t}, t\right\}$, by $N$ particles and their associated importance weights $\left\{{ }_{t}^{(i)}, w_{t}^{(i)}\right\}_{i=1}^{N}$ using the Bayesian Sequential Estimation framework, as follows

$$
\begin{gathered}
{ }_{t}^{(i)} \sim q\left(\left.{ }_{t}\right|_{t-1} ^{(i)}, \mathscr{Y}_{t}\right) \\
w_{t}^{(i)} \quad w_{t-1}^{(i)} \frac{p\left(\mathbf{y}_{t} \mid{ }_{t}^{(i)}\right) p\left(\left.{ }_{t}^{(i)}\right|_{t-1} ^{(i)}\right)}{q\left(\left.{ }_{t}\right|_{t-1} ^{(i)}, \mathscr{Y}_{t}\right)},
\end{gathered}
$$

where $\mathscr{Y}_{t}=\left\{\mathbf{y}_{t^{\prime}}\right\}_{t^{\prime}=t-}^{t}$ with being the width of a sliding window, and ${ }_{i=1}^{N} w_{t}^{(i)}=1$. The proposal function in (5) can be further expanded to

$$
\begin{aligned}
q\left({ }_{t} \mid{ }_{t-1}^{(i)}, \mathscr{Y}_{t}\right)= & q\left(K_{t} \mid K_{t-1}^{(i)}, \mathscr{Y}_{t}\right) q\left(\mathbf{x}_{t} \mid \mathbf{x}_{t-1}^{(i)}, K_{t}^{(i)}, K_{t-1}^{(i)}, \mathscr{Y}_{t}\right) \\
& q\left({ }_{t} \mid \mathbf{x}_{t}^{(i)}, \mathbf{y}_{t}\right),
\end{aligned}
$$

which are the proposal functions for $K_{t}, \mathbf{x}_{t}$, and ${ }_{t}$, respectively.

The set $\left\{\begin{array}{c}(i) \\ t-1\end{array}\right\}_{i=1}^{N}$ and the associated weights $\left\{w_{t-1}^{(i)}\right\}_{i=1}^{N}$ in (6) approximates the posterior distribution of $p\left({ }_{t-1} \mid \mathbf{y}_{1: t-1}\right)$, and $p\left(\mathbf{y}_{t} \mid{ }_{t}{ }^{(i)}\right)$ is the likelihood function. The remaining term in the numerator is the joint conditional prior function for $t$, which has the following hierachical structure

$$
\begin{aligned}
p\left({ }_{t}^{(i)} \mid{ }_{t-1}^{(i)}\right)= & p\left(K_{t}^{(i)} \mid K_{t-1}^{(i)}\right) p\left(\mathbf{x}_{t}^{(i)} \mid \mathbf{x}_{t-1}^{(i)}, K_{t}^{(i)}, K_{t-1}^{(i)}\right) \\
& p\left({ }_{t}^{(i)} \mid \mathbf{x}_{t}^{(i)}, K_{t}^{(i)}\right),
\end{aligned}
$$

which are the prior functions for $K_{t}, \mathbf{x}_{t}$, and ${ }_{t}$, respectively. In the following sections, we provide a brief description of these prior and proposal functions for $t$. Details can be referred to $[3,4]$.

\subsection{Prior functions for $t$}

The prior functions $p\left(\mathbf{x}_{t}^{(i)} \mid \mathbf{x}_{t-1}^{(i)}, K_{t}^{(i)}, K_{t-1}^{(i)}\right)$ and $p\left(K_{t}^{(i)} \mid K_{t-1}^{(i)}\right)$ essentially follow the evolution models of $\mathbf{x}_{t}$ in (1), and $K_{t}$ in (2) and (3), respectively. In this paper, the prior function $p\left({ }_{t}^{(i)} \mid \mathbf{x}_{t}^{(i)}, K_{t}^{(i)}\right)$ takes the similar form as that in [3], but in our approach $K_{t}^{(i)}$ may differ from $K_{t}^{(j)}$, for $i \neq j$.

\subsection{Proposal functions for $t$}

To estimate $K_{t}^{(i)}$, we first search for the regions of interest (ROIs) [3], and hence randomly propose either the birth or death move for track initiation or removal, based on the persistence of these ROIs in representing the true targets. Ultimately, the number of active targets $\hat{K}_{t}$ can be determined by the histogram of the particles $\left\{K_{t}^{(i)}\right\}_{i=1}^{N}$.

\footnotetext{
${ }^{1}$ The notation $(\cdot)_{1: t}$ indicates all the elements from time 1 to time $t$.
}

Given $K_{t}^{(i)}$ and $\mathbf{x}_{t-1}^{(i)}$, we are able to sample the state particles according to $\mathbf{x}_{t}^{(i)} \sim q\left(\mathbf{x}_{t} \mid \mathbf{x}_{t-1}^{(i)}, K_{t}^{(i)}, K_{t-1}^{(i)}, \mathscr{Y}_{t}\right)$. In general, this form is difficult to get, so one may choose the dynamic prior as the proposal function, i.e., $\mathbf{x}_{t}^{(i)} \sim$ $p\left(\mathbf{x}_{t} \mid \mathbf{x}_{t-1}^{(i)}, K_{t}^{(i)}, K_{t-1}^{(i)}\right)$. With the particles $\left\{\mathbf{x}_{t}^{(i)}, K_{t}^{(i)}\right\}$, we may determine the data association vector ${ }_{t}^{(i)}$ using the softgating approach that combines the efficient $\mathfrak{M}$-best 2 -D data assignment algorithm [5,7], which optimally computes all feasible measurement-to-target assignments, subject to certain constraints, with the sampling methods [4]. Once the particles $\left\{{ }_{t}^{(i)}\right\}_{i=1}^{N}$ are available, the associated importance weights $\left\{w_{t}^{(i)}\right\}_{i=1}^{N}$ can be determined according to (6).

\section{BIRTH / DEATH MOVES}

The target detection module in our approach is similar to that in [3], where a clustering algorithm which groups a set of target originating observations within a sliding window plays a key role. The main differences between these two approaches are that in our approach the number of targets is stochastically estimated and the particles $K_{t}^{(i)}$, for $i=1, \ldots, N$, may have different values.

The basic idea of the clustering algorithm is that a set of ROIs within the surveillance region is searched, each of which represents a set of grouped observations that are likely to originate from true targets. It can be shown [3] that there is an association between the true targets and the sets of ROIs representing these targets. The persistences of these associated ROIs provide a clue when targets appear and disappear.

Let $p_{b}^{o}=\left(t-t_{0}^{o}\right) /{ }_{b}$ be a measure of persistence of object $o$ that is being detected by a sequence of regions, where $t_{0}^{o}$ is the time at which the ROI representing the object $o$ is first detected and $b$ is a pre-defined threshold. If object $o$ is a true target, the quantity $p_{b}^{o}$ increases with time; otherwise, the quantity $p_{b}^{o}$ is generally less than 1 . The closer the value of $p_{b}^{o}$ to one, the more certainly target initiation or the birth move should be executed.

For track removal, we may exploit the fact that when a target vanishes, the ROIs representing this target will also vanish with some delay. Let $t_{1}^{k^{\prime}}$ be the last time track $k^{\prime}$ can be associated with a measurement, and $p_{d}^{k^{\prime}}=\left(t-t_{1}^{k^{\prime}}\right) /{ }_{d}$ be a measure of persistence of track $k^{\prime}$ that fails to associate with a measurement, with ${ }_{d}$ being a pre-defined threshold. If track $k^{\prime}$ has persistently failed to associate with a measurement, the quantity $p_{d}^{k^{\prime}}$ increases with time. The closer the value of $p_{d}^{k^{\prime}}$ to one, the more certainty track removal or the death move should be executed. The following schemas summarise the procedures for the birth and death moves, respectively.

\section{Birth Move}

Given a set of ROIs that are unassociated with any existing tracks [3], we first compute the persistence measure $p_{b}^{o}$. For $i=1, \ldots, N$,

- Draw a random sample $u \sim \mathscr{U}_{[0,1]}$.

- If $\min \left(p_{b}^{o}, 1\right) \geq u$, then $K_{t}^{(i)}=K_{t-1}^{(i)}+1$. A unique track ID will be assigned to the object $o$ and maintained throughout its state estimation. 
1. Initialise the state components of the new track using the spatial information given by the associated ROI. For example, we may uniformly sample a measurement within the ROI and convert it to the initial state information for initialisation to yield $\tilde{\mathbf{x}}_{K_{t}^{(i)}}^{(i)}$. For more information about this initialisation procedure, please refer to [3].

2. Sample $\mathbf{x}_{t}^{\prime(i)} \sim q\left(\mathbf{x}_{t}^{\prime} \mid \mathbf{x}_{t-1}^{(i)}, K_{t-1}^{(i)}\right)$.

3. Append the initialised state component $\tilde{\mathbf{x}}_{K_{t}^{(i)}}^{(i)}$ with $\mathbf{x}_{t}^{\prime(i)}$, yielding $\mathbf{x}_{t}^{(i)}=\left[\mathbf{x}_{t}^{\prime(i)} \mid \tilde{\mathbf{x}}_{K_{t}^{(i)}}^{(i)}\right]$

- else $K_{t}^{(i)}=K_{t-1}^{(i)}$.

1. Sample $\mathbf{x}_{t}^{(i)} \sim q\left(\mathbf{x}_{t} \mid \mathbf{x}_{t-1}^{(i)}, K_{t}^{(i)}\right)$.

- Compute the data association vector ${ }_{t}^{(i)}$, given $\mathbf{y}_{t}, \mathbf{x}_{t}^{(i)}$, and $K_{t}^{(i)}$, using the proposed approach.

- Update the importance weight $w_{t}^{(i)}$ according to (6)

- Repeat the procedure for $i \rightarrow i+1$.

\section{Death Move}

For $i=1, \ldots, N$, compute $p_{d}^{k}$ for all active tracks for $k=$ $1, \ldots, K_{t-1}^{(i)}$.

- Randomly select one track for removal, say $k^{\prime} \in$ $\left\{1, \ldots, K_{t}^{(i)}\right\}$, given $p_{d}^{k^{\prime}}>0$.

- Draw a random sample $u \sim \mathscr{U}_{[0,1]}$.

- If $\min \left(p_{d}^{k^{\prime}}, 1\right) \geq u$, then $K_{t}^{(i)}=K_{t-1}^{(i)}-1$, and its track ID will be deleted.

1. Remove the components of track $k^{\prime}$ from the combined state vector $\mathbf{x}_{t-1}^{(i)}$. That is, $\tilde{\mathbf{x}}_{t-1}^{(i)}=$ $\left[\mathbf{x}_{1, t-1}^{(i)^{T}}, \ldots, \mathbf{x}_{k^{\prime}-1, t-1}^{(i)^{T}}, \mathbf{x}_{k^{\prime}+1, t-1}^{(i)}, \ldots, \mathbf{x}_{K_{t-1}^{(i)}, t-1}^{(i)}\right]^{T}$.

2. Sample $\mathbf{x}_{t}^{(i)} \sim q\left(\mathbf{x}_{t} \mid \tilde{\mathbf{x}}_{t-1}^{(i)}, K_{t}^{(i)}\right)$.

- else $K_{t}^{(i)}=K_{t-1}^{(i)}$.

1. Sample $\mathbf{x}_{t}^{(i)} \sim q\left(\mathbf{x}_{t} \mid \mathbf{x}_{t-1}^{(i)}, K_{t}^{(i)}\right)$.

- Compute the data association vector ${ }_{t}^{(i)}$, given $\mathbf{y}_{t}, \mathbf{x}_{t}^{(i)}$ and $K_{t}^{(i)}$, using the proposed approach.

- Update the importance weight $w_{t}^{(i)}$ according to (6).

- Repeat the procedure for $i \rightarrow i+1$.

\section{COMPUTER SIMULATIONS}

In this section, we examine the performance of the proposed algorithm in the following areas: target detection, tracking, and data association, using a single sensor, located at $\left(x_{o}, y_{o}\right)=(0,0)$. The environment in the experiment is very hostile in which the target detection probability $P_{D}=0.5$ and the clutter rate is $C=20$. The surveillance region $\mathscr{R}_{V}$ is $[2000,2000]^{2}$. Fig. 1 shows all observations for $T=1000$ scans, including target and clutter measurements. Moreover,

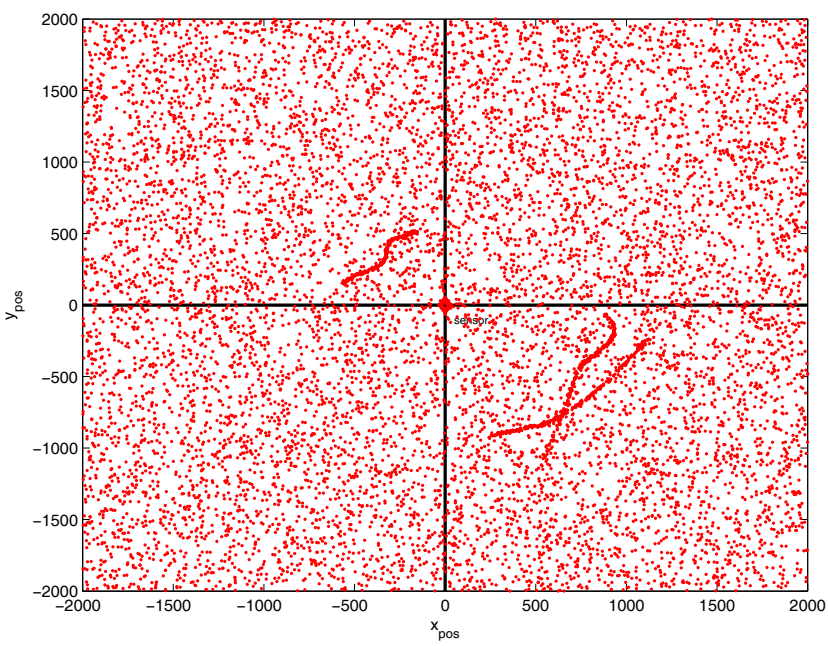

Figure 1: A plot of all measurements for $T=1000$ scans.

\begin{tabular}{c|c}
\hline Parameters & Values \\
\hline $\boldsymbol{\Sigma}_{c v}$ & $\operatorname{diag}\left(\left[5 \times 10^{-4}, 5 \times 10^{-4}\right]\right)$ \\
$\Sigma_{w}$ & $\operatorname{diag}([0.0001,25])$ \\
$b, \quad d$ & 5,10 \\
\hline
\end{tabular}

Table 1: Parameters for computer simulation.

the observation model we adopt is a nonlinear model and contains two components - bearing and range. Three targets are synthesised according to the nearly constant velocity model [5] with parameters summarised in Table 1. The state and observation noises are assumed Gaussian random variables with zero mean and covariance matrices, $\boldsymbol{\Sigma}_{c v}$ and $\boldsymbol{\Sigma}_{w}$, respectively. A total of 1000 particles are used to estimate the posterior distribution function $p\left({ }_{t} \mid \mathbf{y}_{1: t}\right)$. According to Fig. 2, all tracks are well detected as described in Section 3, except the time delays incurred when initiating and removing tracks. Figs. 3 and 4 compare the true tracks and their estimates. It can be seen that not only are the targets well tracked, but the measurement-to-target association using the soft-gating method is also done well.

Finally, the tracking performance of the proposed method is evaluated as a function of different numbers of particles $N$ in terms of the Root Mean Square Error (RMSE), defined as

$$
R M S E_{l}=\sqrt{\frac{1}{K(t) T}}{ }_{t=1}^{T}\left\|\mathbf{x}_{t}-\hat{\mathbf{x}}_{t}^{l}(N)\right\|^{2},
$$

\begin{tabular}{c|c|c}
\hline $\mathbf{N}$ & RMSE & \\
\hline 500 & 22.5 & 4.3 \\
1000 & 20.1 & 3.0 \\
1500 & 14.4 & 2.4 \\
2000 & 13.2 & 2.2 \\
5000 & 12.9 & 1.8 \\
\hline
\end{tabular}

Table 2: Evaluation of the tracking performance of the proposed algorithm for different values of $N$ in 20 independent runs. 


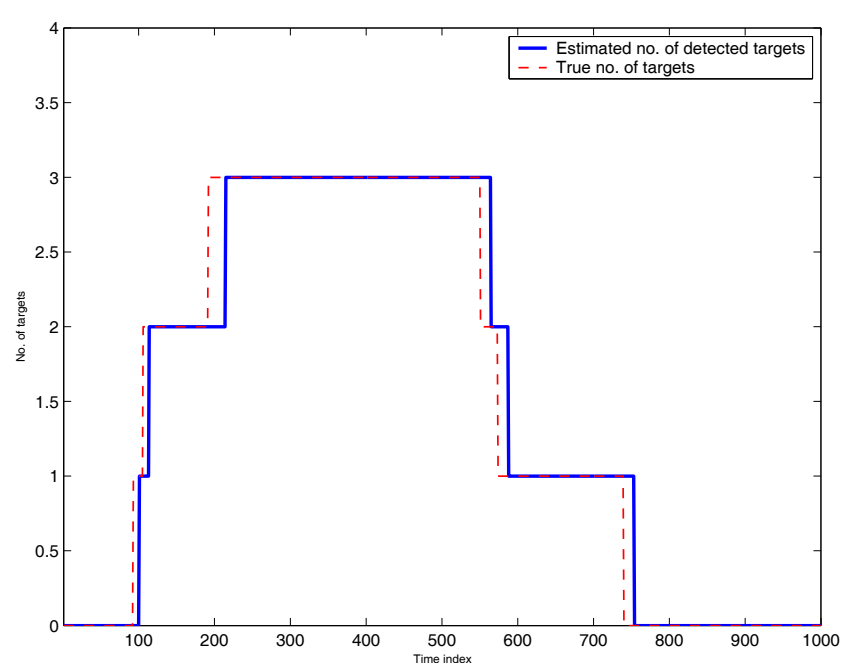

Figure 2: The trajectories of the online detection of number of targets.

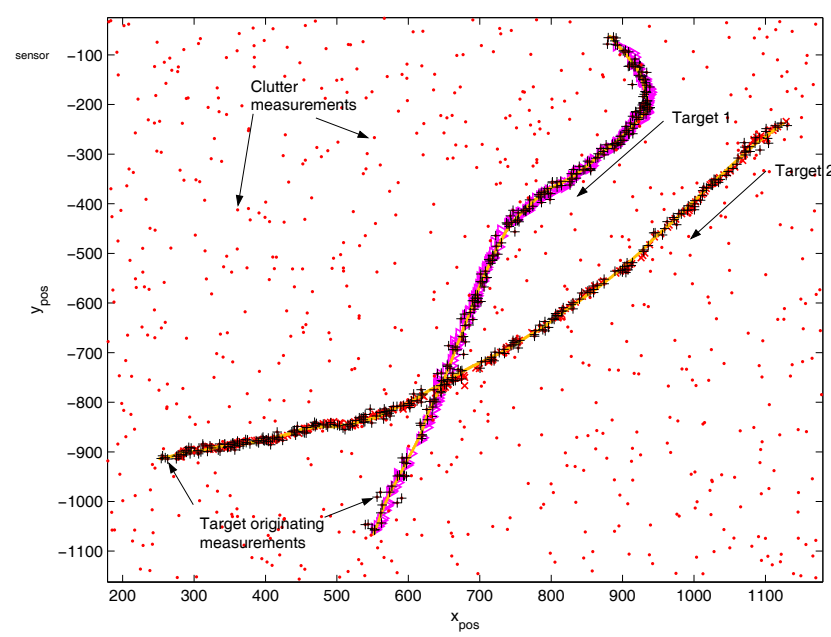

Figure 3: A comparison between Targets 1 and 2 and their estimates.

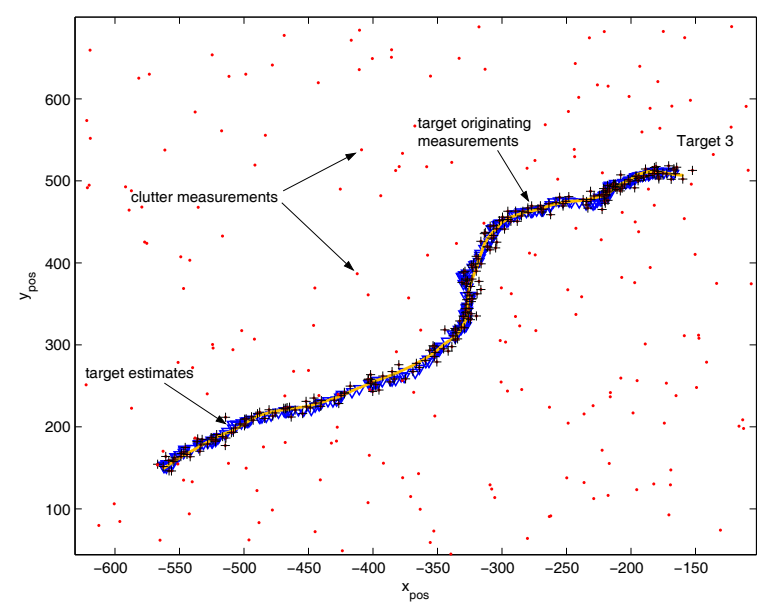

Figure 4: A comparison between Target 3 and its estimates. where $R M S E_{l}$ is the error for the $l$ th independent run, and $\hat{\mathbf{x}}_{t}^{l}(N)$ is a posterior mean estimate of $\mathbf{x}_{t}$ for $l$ th run with $N$ particles. For each value of $N$, a total of 20 independent runs were used with the same synthesised tracks but different observations, and the RMSEs and the standard deviations are shown in Table 2. As expected, the RMSE decreases as $N$ increases, at the expense of an increased computational load.

\section{CONCLUSIONS}

In this paper, we presented a new approach for joint detection and tracking for multiple targets using sequential Monte Carlo methods. Using the information from the detected ROIs by the clustering algorithm, we may estimate the number of targets by proposing either the birth or death move, followed by the target state estimation and measurement-totarget association using the soft-gating approach. Computer simulations demonstrated that the proposed approach is very robust in estimating the number of targets, even though the environment is very hostile in terms of high clutter density and low target detection probability.

\section{ACKNOWLEDGEMENTS}

The research of $\mathrm{Ng}, \mathrm{Li}$ and Godsill was sponsored by the Data and Information Fusion - Defence Technology Centre, UK, Research Grant 10.2. Vermaak's work was funded by QinetiQ under contract QinetiQ Contract CU006 0000014890 . The authors thank these parties for funding this work. Furthermore, the authors appreciate the valuable input from S. Maskell and M. Briers in QinetiQ, UK, for sharing the code of the auction algorithm for measurement-to-target assignment.

\section{REFERENCES}

[1] H. Sidenbladh, "Multi-target particle filtering for the probability hypothesis density," Proceedings 6th International Conference on Information Fusion, pp. 800 806, 2003.

[2] H. Sidenbladh and S. L. Wirkander, "Particle filtering for finite random sets," IEEE Transactions on Aerospace and Electronic Systems, 2003. to appear.

[3] W. Ng, J. Li, S. Godsill, and J. Vermaak, "A hybrid approach for online joint detection and tracking for multiple targets," in Proceedings of the IEEE Aerospace Conference 2005, 2005. Accepted by the IEEE Aerospace Conference 2005. See http://wwwsigproc.eng.cam.ac.uk/ kfn20/.

[4] W. Ng, J. Li, S. Godsill, and J. Vermaak, "Multiple target tracking using a new soft-gating approach and sequential Monte Carlo methods," in Proceedings of the International Conference on Acoustics, Speech, and Signal Processing, vol. 4, pp. 1049-1052, 2005.

[5] Y. Bar-Shalom and T. E. Fortmann, Tracking and Data Association. Academic Press, 1988.

[6] Y. Bar-Shalom and W. D. Blair, Multitarget-Multisensor Tracking: Applications and Advances, vol. III. Norwood, MS: Archtech House, 2000.

[7] S. Blackman and R. Popoli, Design and Analysis of Modern Tracking Systems. Norwood, MA: Artech House, 1999. 